\title{
Strange Duality Between Hypersurface and Complete Intersection Singularities
}

\author{
Wolfgang Ebeling ${ }^{1} \cdot$ Atsushi Takahashi $^{2}$
}

Received: 22 September 2015 / Revised: 9 May 2016 / Accepted: 12 May 2016 /

Published online: 24 May 2016

(C) Institute for Mathematical Sciences (IMS), Stony Brook University, NY 2016

\begin{abstract}
W. Ebeling and C. T. C. Wall discovered an extension of Arnold's strange duality embracing on one hand series of bimodal hypersurface singularities and on the other, isolated complete intersection singularities. In this paper, we derive this duality from the mirror symmetry and the Berglund-Hübsch transposition of invertible polynomials.
\end{abstract}

Keywords Mirror symmetry $\cdot$ Singularity $\cdot$ Invertible polynomial $\cdot$ Strange duality Dolgachev numbers · Gabrielov numbers

Mathematics Subject Classification $14 \mathrm{~J} 33 \cdot 32 \mathrm{~S} 20 \cdot 32 \mathrm{~S} 30 \cdot 14 \mathrm{~L} 30$

\section{Introduction}

During his classification of hypersurface singularities, Arnold (1975) observed a strange duality between the 14 exceptional unimodal singularities. Ebeling and Wall (1985) discovered an extension of this duality embracing on one hand series of bimodal

Partially supported by the DFG-programme SPP1388 "Representation Theory" and by JSPS KAKENHI Grant Numbers 24684005 and 16H02146.

Wolfgang Ebeling

ebeling@math.uni-hannover.de

Atsushi Takahashi

takahashi@math.sci.osaka-u.ac.jp

1 Institut für Algebraische Geometrie, Leibniz Universität Hannover, Postfach 6009, 30060 Hannover, Germany

2 Department of Mathematics, Graduate School of Science, Osaka University, Toyonaka, Osaka 560-0043, Japan 
singularities and on the other, isolated complete intersection singularities (ICIS) in $\mathbb{C}^{4}$. The duals of the complete intersection singularities are not themselves singularities, but are virtual $(k=-1)$ cases of series (e.g. $\left.W_{1, k}: k \geq 0\right)$ of bimodal singularities. They associated to these well-defined Coxeter-Dynkin diagrams and Milnor lattices and showed that all numerical features of Arnold's strange duality continue to hold. The $k=-1$ cases of the series were called virtual singularities in Ebeling and Wall (1985), because setting $k=-1$ in Arnold's equations of the series one gets exceptional unimodal singularities with a smaller Milnor number as germs at the origin.

The objective of this paper is to derive this extended strange duality from the mirror symmetry and the Berglund-Hübsch transposition of invertible polynomials. Moreover, we show that the virtual singularities exist in the sense that the equations have to be considered as global polynomials. The bimodal series start with singularities with $k=0$ (e.g. $W_{1,0}$ ). They can be given by polynomials with two moduli. Setting one of the moduli equal to zero, one is left with a one-parameter family of weighted homogeneous polynomials. It is natural from the mirror symmetry view point to expect that adding one monomial to an invertible polynomial is dual to having another $\mathbb{C}^{*}$ action on the dual polynomial, which leads to our duality between virtual singularities and complete intersection singularities.

We shall proceed as follows. We first classify the non-degenerate invertible polynomials with a $\mathbb{Z} / 2 \mathbb{Z}$-action. They are defined by certain $3 \times 3$-matrices. Then we shall classify the possibilities to extend such a $3 \times 3$-matrix to a certain $4 \times 3$-matrix satisfying certain conditions. Such a matrix defines a polynomial $\mathbf{f}(x, y, z)$ with four monomials with a non-isolated singularity. We shall consider the Berglund-Hübsch transpose of this polynomial. The kernel of the transpose $3 \times 4$-matrix defines a $\mathbb{C}^{*}$ action on the space $\mathbb{C}^{4}$ and this matrix and the degree 0 polynomials define a complete intersection singularity in $\mathbb{C}^{4}$ as the zero set of two polynomials.

Following Ebeling and Takahashi (2011), we consider the polynomial $\mathbf{f}(x, y, z)-$ $x y z$. Under certain conditions, there is a coordinate transformation which transforms this polynomial to a polynomial $\mathbf{h}(x, y, z)-x y z$, where $\mathbf{h}$ is again a polynomial with four monomials, but now has an isolated singularity at the origin. We call this a virtual singularity. The polynomial $\mathbf{h}$ is no longer weighted homogeneous but its Newton polygon at infinity has two two-dimensional faces. We thus obtain a duality between the virtual hypersurface singularities and complete intersection singularities.

We show that this duality has the features of Arnold's strange duality. Namely, we associate Dolgachev and Gabrielov numbers to the polynomials $\mathbf{h}$ and the equations defining the complete intersection singularities generalizing the approach of Ebeling and Takahashi (2011). It turns out that the Dolgachev numbers of the polynomial $\mathbf{h}$ are the Gabrielov numbers of the pair of polynomials defining the complete intersection singularity and vice versa, the Gabrielov numbers of the polynomial $\mathbf{h}$ are the Dolgachev numbers of the pair of polynomials defining the complete intersection singularity. Moreover, we show that the reduced zeta function of the monodromy at infinity of a virtual singularity coincides with the product of the Poincare series of the coordinate ring of the dual complete intersection singularity and a polynomial encoding its Dolgachev numbers.

As an example we consider those singularities with Gorenstein parameter being equal to 1 . In this way, we recover precisely the virtual singularities of the bimodal 
series and the extension of Arnold's strange duality found in Ebeling and Wall (1985). Therefore we have shown that these virtual singularities exist as global polynomials. Moreover, the Dolgachev and Gabrielov numbers which we have associated to them agree with the ones predicted in Ebeling and Wall (1985). Finally, we construct Coxeter-Dynkin diagrams for the virtual bimodal singularities and show that they can be transformed to graphs which have the same shape as in the exceptional unimodal case used in Gabrielov's original definition of the numbers now named after him.

\section{Invertible Polynomials}

We recall some general definitions about invertible polynomials.

Let $f\left(x_{1}, \ldots, x_{n}\right)$ be a weighted homogeneous polynomial, namely, a polynomial with the property that there are positive integers $w_{1}, \ldots, w_{n}$ and $d$ such that $f\left(\lambda^{w_{1}} x_{1}, \ldots, \lambda^{w_{n}} x_{n}\right)=\lambda^{d} f\left(x_{1}, \ldots, x_{n}\right)$ for $\lambda \in \mathbb{C}^{*}$. We call $\left(w_{1}, \ldots, w_{n} ; d\right)$ a system of weights.

Definition A weighted homogeneous polynomial $f\left(x_{1}, \ldots, x_{n}\right)$ is called invertible if the following conditions are satisfied:

(i) the number of variables $(=n)$ coincides with the number of monomials in the polynomial $f\left(x_{1}, \ldots, x_{n}\right)$, namely,

$$
f\left(x_{1}, \ldots, x_{n}\right)=\sum_{i=1}^{n} a_{i} \prod_{j=1}^{n} x_{j}^{E_{i j}}
$$

for some coefficients $a_{i} \in \mathbb{C}^{*}$ and non-negative integers $E_{i j}$ for $i, j=1, \ldots, n$, (ii) a system of weights $\left(w_{1}, \ldots, w_{n} ; d\right)$ can be uniquely determined by the polynomial $f\left(x_{1}, \ldots, x_{n}\right)$ up to a constant factor $\operatorname{gcd}\left(w_{1}, \ldots, w_{n} ; d\right)$, namely, the matrix $E:=\left(E_{i j}\right)$ is invertible over $\mathbb{Q}$.

An invertible polynomial is called non-degenerate, if it has an isolated singularity at the origin.

Without loss of generality one may assume that $a_{i}=1$ for $i=1, \ldots, n$. This can be achieved by rescaling the variables. We may and shall also assume that $\operatorname{det} E>0$.

An invertible polynomial has a canonical system of weights $W_{f}=\left(w_{1}, \ldots, w_{n} ; d\right)$ given by the unique solution of the equation

$$
E\left(\begin{array}{l}
w_{1} \\
\vdots \\
w_{n}
\end{array}\right)=\operatorname{det}(E)\left(\begin{array}{l}
1 \\
\vdots \\
1
\end{array}\right), \quad d:=\operatorname{det}(E)
$$

This system of weights is in general non-reduced, i.e. in general $c_{f}:=\operatorname{gcd}\left(w_{1}, \ldots\right.$, $\left.w_{n}, d\right)>1$. 
Definition Let $f\left(x_{1}, \ldots, x_{n}\right)=\sum_{i=1}^{n} a_{i} \prod_{j=1}^{n} x_{j}^{E_{i j}}$ be an invertible polynomial. Consider the free abelian group $\oplus_{i=1}^{n} \mathbb{Z} \vec{x}_{i} \oplus \mathbb{Z} \vec{f}$ generated by the symbols $\vec{x}_{i}$ for the variables $x_{i}$ for $i=1, \ldots, n$ and the symbol $\vec{f}$ for the polynomial $f$. The maximal grading $L_{f}$ of the invertible polynomial $f$ is the abelian group defined by the quotient

$$
L_{f}:=\bigoplus_{i=1}^{n} \mathbb{Z} \vec{x}_{i} \oplus \mathbb{Z} \vec{f} / I_{f}
$$

where $I_{f}$ is the subgroup generated by the elements

$$
\vec{f}-\sum_{j=1}^{n} E_{i j} \vec{x}_{j}, \quad i=1, \ldots, n
$$

Definition Let $f\left(x_{1}, \ldots, x_{n}\right)$ be an invertible polynomial and $L_{f}$ be the maximal grading of $f$. The maximal abelian symmetry group $\widehat{G}_{f}$ of $f$ is the abelian group defined by

$$
\widehat{G}_{f}:=\operatorname{Spec}\left(\mathbb{C} L_{f}\right),
$$

where $\mathbb{C} L_{f}$ denotes the group ring of $L_{f}$. Equivalently,

$$
\widehat{G}_{f}=\left\{\left(\lambda_{1}, \ldots, \lambda_{n}\right) \in\left(\mathbb{C}^{*}\right)^{n} \mid \prod_{j=1}^{n} \lambda_{j}^{E_{1 j}}=\cdots=\prod_{j=1}^{n} \lambda_{j}^{E_{n j}}\right\} .
$$

Moreover, we define

$$
G_{f}=\left\{\left(\lambda_{1}, \ldots, \lambda_{n}\right) \in \widehat{G}_{f} \mid \prod_{j=1}^{n} \lambda_{j}^{E_{1 j}}=\cdots=\prod_{j=1}^{n} \lambda_{j}^{E_{n j}}=1\right\} .
$$

Let $f\left(x_{1}, \ldots, x_{n}\right)$ be an invertible polynomial and $W_{f}=\left(w_{1}, \ldots, w_{n} ; d\right)$ be the canonical system of weights associated to $f$. Set

$$
q_{i}:=\frac{w_{i}}{d}, \quad i=1, \ldots, n
$$

Note that $G_{f}$ always contains the exponential grading operator

$$
g_{0}:=\left(\exp \left(2 \pi \sqrt{-1} q_{1}\right), \ldots, \exp \left(2 \pi \sqrt{-1} q_{n}\right)\right)
$$

Let $G_{0}$ be the subgroup of $G_{f}$ generated by $g_{0}$. One has (cf. Ebeling and Takahashi 2013)

$$
\left[G_{f}: G_{0}\right]=c_{f}
$$


Let $f\left(x_{1}, \ldots, x_{n}\right)=\sum_{i=1}^{n} a_{i} \prod_{j=1}^{n} x_{j}^{E_{i j}}$ be an invertible polynomial. Following Berglund and Hübsch (1993), the Berglund-Hübsch transpose $\tilde{f}\left(x_{1}, \ldots, x_{n}\right)$ of $f$ is defined by

$$
\widetilde{f}\left(x_{1}, \ldots, x_{n}\right)=\sum_{i=1}^{n} a_{i} \prod_{j=1}^{n} x_{j}^{E_{j i}} .
$$

By Berglund and Henningson (1995), for a subgroup $G \subset G_{f}$ its dual group $\widetilde{G}$ is defined by

$$
\widetilde{G}:=\operatorname{Hom}\left(G_{f} / G, \mathbb{C}^{*}\right) .
$$

Note that $\operatorname{Hom}\left(G_{f}, \mathbb{C}^{*}\right)$ is isomorphic to $G_{\tilde{f}}$, see Berglund and Henningson (1995). By Krawitz (2009), we have

$$
\widetilde{G}_{0}=\mathrm{SL}_{n}(\mathbb{Z}) \cap G_{\widetilde{f}}
$$

Moreover, by Ebeling and Takahashi (2013, Proposition 3.1), we have $\left|\widetilde{G}_{0}\right|=c_{f}$.

\section{Invertible Polynomials with $\mathbb{Z} / 2 \mathbb{Z}$-Action}

Let $f(x, y, z)$ be a non-degenerate invertible polynomial with $\left[G_{f}: G_{0}\right]=2$. We shall now classify the non-degenerate invertible polynomials with such a group action.

Proposition 1 There are the following non-degenerate invertible polynomials $f(x, y$, $z)$ with $\left[G_{f}: G_{0}\right]=2$. We list the possible types and the conditions. The coordinates are chosen so that the action of $\widetilde{G}_{0}=\mathbb{Z} / 2 \mathbb{Z}$ on $\widetilde{f}$ is given by $(x, y, z) \mapsto(-x,-y, z)$.

I: $f(x, y, z)=x^{p_{1}}+y^{p_{2}}+z^{p_{3}} ; p_{1}, p_{2}$ even,

IIA: $f(x, y, z)=x^{p_{2}}+x y^{p_{3} / p_{2}}+z^{p_{1}} ; p_{2}$ odd,$p_{3} / p_{2}$ even,

IIB: $f(x, y, z)=x^{p_{1}}+y^{p_{2}}+y z^{p_{3} / p_{2}} ; p_{1}, p_{2}$ even,

III: $f(x, y, z)=x^{q_{2}+1} y+x y^{q_{3}+1}+z^{p_{1}} ; q_{2}, q_{3}$ even,

IV: $f(x, y, z)=x^{p_{1}}+x y^{\frac{p_{2}}{p_{1}}}+y z^{\frac{p_{3}}{p_{2}}} ; p_{2} / p_{1}$ even, $p_{1}$ odd.

Proof This follows by inspection of Ebeling and Takahashi (2011, Table 1).

Let $\widehat{G}_{0}$ be the subgroup of $\widehat{G}_{f}$ defined by the commutative diagram of short exact sequences

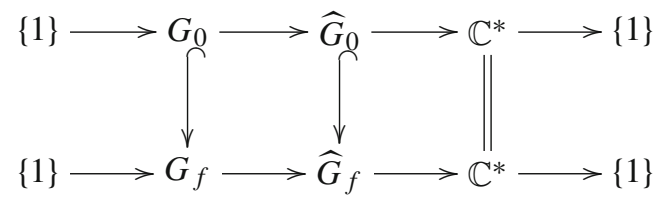

Let $L_{0}$ be the quotient of $L_{f}$ corresponding to the subgroup $\widehat{G}_{0}$ of $\widehat{G}_{f}$. 
We shall now classify $4 \times 3$-matrices $E=\left(E_{i j}\right)_{j=1,2,3}^{i=1,2,3,4}$ such that

$$
\mathbb{Z} \vec{x} \oplus \mathbb{Z} \vec{y} \oplus \mathbb{Z} \vec{z} \oplus \mathbb{Z} \vec{f} /\left\langle E_{i 1} \vec{x}+E_{i 2} \vec{y}+E_{i 3} \vec{z}=\vec{f}, i=1, \ldots, 4\right\rangle \cong L_{0}
$$

and $\mathcal{C}_{\left(F, G_{0}\right)}:=\left[\left(F^{-1}(0) \backslash\{0\}\right) / \widehat{G}_{0}\right]$, where $F:=\sum_{i=1}^{4} a_{i} x^{E_{i 1}} y^{E_{i 2}} z^{E_{i 3}}$, is a smooth projective line with 4 isotropic points whose orders are $\alpha_{1}, \alpha_{2}, \alpha_{3}, \alpha_{4}$, where $A_{\left(f, G_{0}\right)}=$ $\left(\alpha_{1}, \alpha_{2}, \alpha_{3}, \alpha_{4}\right)$ are the Dolgachev numbers of the pair $\left(f, G_{0}\right)$ defined in Ebeling and Takahashi (2013), for general $a_{1}, a_{2}, a_{3}, a_{4}$.

Proposition 2 The possible matrices $E$ are classified into the following types up to a permutation of the rows. The matrices are described by the corresponding polynomials $F$.

$\mathrm{I}: a_{1} x^{p_{1}}+a_{2} y^{p_{2}}+a_{3} z^{p_{3}}+a_{4} x^{\frac{p_{1}}{2}} y^{\frac{p_{2}}{2}}$

IIA: $a_{1} x^{p_{2}}+a_{2} x y^{\frac{p_{3}}{p_{2}}}+a_{3} z^{p_{1}}+a_{4} x^{\frac{p_{2}+1}{2}} y^{\frac{p_{3}}{2 p_{2}}}$

IIB: $a_{1} x^{p_{1}}+a_{2} y^{p_{2}}+a_{3} y z^{\frac{p_{3}}{p_{2}}}+a_{4} x^{\frac{p_{1}}{2}} y^{\frac{p_{2}}{2}}$

$\mathrm{IIB}^{\sharp}:\left(p_{2}=2\right) a_{1} x^{\frac{p_{1}}{2}} z^{\frac{p_{3}}{2}}+a_{2} y^{2}+a_{3} y z^{\frac{p_{3}}{2}}+a_{4} x^{\frac{p_{1}}{2}} y$

III: $a_{1} x^{q_{2}+1} y+a_{2} x y^{q_{3}+1}+a_{3} z^{p_{1}}+a_{4} x^{\frac{q_{2}}{2}+1} y^{\frac{q_{3}}{2}+1}$

$\mathrm{IV}: a_{1} x^{p_{1}}+a_{2} x y^{\frac{p_{2}}{p_{1}}}+a_{3} y z^{\frac{p_{3}}{p_{2}}}+a_{4} x^{\frac{p_{1}+1}{2}} y^{\frac{p_{2}}{2 p_{1}}}$

$\mathrm{IV}^{\sharp}:\left(\frac{p_{2}}{p_{1}}=2\right) a_{1} x^{\frac{p_{1}-1}{2}} z^{\frac{p_{3}}{p_{2}}}+a_{2} x y^{2}+a_{3} y z^{\frac{p_{3}}{p_{2}}}+a_{4} x^{\frac{p_{1}+1}{2}} y$

Proof We only give the proof for the case IIB which is the most difficult one. The other cases are easier and are treated analogously.

In the case IIB, the group $L_{f}$ is the quotient of the abelian group $\mathbb{Z} \vec{x} \oplus \mathbb{Z} \vec{y} \oplus \mathbb{Z} \vec{z} \oplus \mathbb{Z} \vec{f}$ given by the relations $p_{1} \vec{x}=p_{2} \vec{y}=\vec{y}+\frac{p_{3}}{p_{2}} \vec{z}=\vec{f}$ and $L_{0}$ is given by the additional relation

$$
\frac{p_{1}}{2} \vec{x}=\frac{p_{2}}{2} \vec{y}
$$

We derive from these relations the relation

$$
\left(p_{2}-1\right) \vec{y}=\frac{p_{3}}{p_{2}} \vec{z}
$$

1. We first classify all monomials which can appear in $F$. Suppose a monomial $x^{a} y^{b}$ appears. Then we must have

$$
a \vec{x}+b \vec{y}=\vec{f}=\frac{p_{1}}{2} \vec{x}+\frac{p_{2}}{2} \vec{y} .
$$

From this we get

$$
\left(\frac{p_{1}}{2}-a\right) \vec{x}=\left(b-\frac{p_{2}}{2}\right) \vec{y} .
$$

By relation (3.1) there must exist an integer $c$ such that

$$
\frac{p_{1}}{2}-a=\frac{p_{1}}{2} c \text { and } b-\frac{p_{2}}{2}=\frac{p_{2}}{2} c .
$$


But

$$
\frac{p_{1}}{2}(1-c)=a \geq 0 \text { and } \frac{p_{2}}{2}(c+1)=b \geq 0 .
$$

This implies $c=-1,0,1$. Therefore we obtain the possibilities

$$
x^{\frac{p_{1}}{2}} y^{\frac{p_{2}}{2}}, y^{p_{2}} \text { or } x^{p_{1}} .
$$

In a similar way, using the relation (3.2), we can derive the possibilities

$$
y^{p_{2}}, y z^{\frac{p_{3}}{p_{2}}} \text { or } z^{p_{3}} \quad\left(\text { if } p_{2}=2\right) \text {. }
$$

Now suppose that a monomial $x^{a} z^{b}$ appears. Then

$$
a \vec{x}+b \vec{z}=\vec{f}=\vec{y}+\frac{p_{3}}{p_{2}} \vec{z}
$$

From this it follows that $\vec{y}=c \vec{x}$ for some positive integer $c$ since $\vec{y}+\frac{p_{3}}{p_{2}} \vec{z}=\vec{f}$ is the only relation involving $\vec{z}$. Relation (3.1) implies $p_{2}=2, \vec{y}=\frac{p_{1}}{2} \vec{x}$ and

$$
a \vec{x}+b \vec{z}=\frac{p_{1}}{2} \vec{x}+\frac{p_{3}}{2} \vec{z}
$$

This yields the possibilities

$$
x^{p_{1}}, x^{\frac{p_{1}}{2}} z^{\frac{p_{3}}{2}} \quad\left(\text { if } p_{2}=2\right) \quad \text { or } \quad z^{p_{3}} \quad\left(\text { if } p_{2}=2\right) \text {. }
$$

Finally one can derive that there are no monomials of the form $x^{a} y^{b} z^{c}$ with $a, b, c>0$. 2 . Therefore, if $p_{2} \neq 2$, we only obtain the possibility

$$
F(x, y, z)=a_{1} x^{p_{1}}+a_{2} y^{p_{2}}+a_{3} y z^{\frac{p_{3}}{p_{2}}}+a_{4} x^{\frac{p_{1}}{2}} y^{\frac{p_{2}}{2}} .
$$

If $p_{2}=2$ we obtain several possibilities. In this case we have to consider the system of weights for $G_{0}$

$$
\left(\frac{p_{3}}{2}, \frac{p_{1} p_{3}}{4}, \frac{p_{1}}{2} ; \frac{p_{1} p_{3}}{2}\right)
$$

and the Dolgachev numbers of the pair $\left(f, G_{0}\right)$ given by Ebeling and Takahashi (2013)

$$
A_{\left(f, G_{0}\right)}=\left(\frac{p_{1}}{2}, \frac{p_{3}}{2}, \frac{p_{3}}{2}, \frac{p_{1}}{2}\right)
$$

In order to obtain the same Dolgachev numbers for $F, F(1,0, z)$ must be non-zero if $z \neq 0$. Therefore the polynomial $F$ must contain 3 monomials involving $y$. Note here that $L_{0}$ is given by the relations $\left(p_{1} / 2\right) \vec{x}=\vec{y}=\left(p_{3} / 2\right) \vec{z}$ and $2 \vec{y}=\vec{f}$, which 
are symmetric under the change $\vec{x}, p_{1}$ to $\vec{z}, p_{3}$. This leaves us with the only additional possibility

$$
F(x, y, z)=a_{1} x^{\frac{p_{1}}{2}} z^{\frac{p_{3}}{2}}+a_{2} y^{2}+a_{3} y z^{\frac{p_{3}}{2}}+a_{4} x^{\frac{p_{1}}{2}} y .
$$

We associate to these matrices a pair of polynomials as follows. We observe that the kernel of the matrix $E^{T}$ is either generated by the vector $(1,1,0,-2)^{T}$ or by the vector $(1,1,-1,-1)^{T}$. The second case occurs precisely for the matrices of type IIB $\sharp$ and IV $\mathrm{IV}^{\sharp}$. Let $R:=\mathbb{C}[x, y, z, w]$. In the first case, there exists a $\mathbb{Z}$-graded structure on $R$ given by the $\mathbb{C}^{*}$-action

$$
\lambda *(x, y, z, w)=\left(\lambda x, \lambda y, z, \lambda^{-2} w\right) \text { for } \lambda \in \mathbb{C}^{*} .
$$

In the second case, there exists a $\mathbb{Z}$-graded structure on $R$ given by the $\mathbb{C}^{*}$-action

$$
\lambda *(x, y, z, w)=\left(\lambda x, \lambda y, \lambda^{-1} z, \lambda^{-1} w\right) \text { for } \lambda \in \mathbb{C}^{*} .
$$

Let $R=\bigoplus_{i \in \mathbb{Z}} R_{i}$ be the decomposition of $R$ according to one of these $\mathbb{Z}$-gradings. Let $E^{T}$ be the transposed matrix. We associate to this the polynomial

$$
\widetilde{f}(x, y, z, w):=x^{E_{11}} y^{E_{21}} z^{E_{31}} w^{E_{41}}+x^{E_{12}} y^{E_{22}} z^{E_{32}} w^{E_{42}}+x^{E_{13}} y^{E_{23}} z^{E_{33}} w^{E_{43}} .
$$

In the first case, we have $\widetilde{f} \in R_{0}=\mathbb{C}\left[x^{2} w, y^{2} w, z, x y w\right]$. Let

$$
X:=x^{2} w, \quad Y:=y^{2} w, \quad Z:=z, \quad W:=x y w .
$$

In these new coordinates, we obtain a pair of polynomials

$$
\tilde{\mathbf{f}}_{1}(X, Y, Z, W)=X Y-W^{2}, \quad \widetilde{\mathbf{f}}_{2}(X, Y, Z, W)=\widetilde{f}(X, Y, Z, W) .
$$

In the second case, we have $\tilde{f} \in R_{0}=\mathbb{C}[x w, y z, x z, y w]$. Let

$$
X:=x w, \quad Y:=y z, \quad Z:=x z \quad W:=y w .
$$

In these new coordinates, we obtain a pair of polynomials

$$
\widetilde{\mathbf{f}}_{1}(X, Y, Z, W)=X Y-Z W, \quad \widetilde{\mathbf{f}}_{2}(X, Y, Z, W)=\widetilde{f}(X, Y, Z, W) .
$$

Now we choose for each of the matrices $E$ special values $a_{1}, a_{2}, a_{3}, a_{4}$ such that the corresponding polynomial $F$ has a non-isolated singularity. We denote this polynomial by $\mathbf{f}$. We summarize the results in Table 1 . 
Table 1 Correspondence between polynomials $\mathbf{f}$ and pairs of polynomials $\left(\widetilde{\mathbf{f}}_{1}, \widetilde{\mathbf{f}}_{2}\right)$

\begin{tabular}{|c|c|c|}
\hline Type & f & $\left(\widetilde{\mathbf{f}}_{1}, \widetilde{\mathbf{f}}_{2}\right)$ \\
\hline I & $x^{p_{1}}+y^{p_{2}}+z^{p_{3}}-2 x^{\frac{p_{1}}{2}} y^{\frac{p_{2}}{2}}$ & $\left\{\begin{array}{c}X Y-W^{2} \\
X^{\frac{p_{1}}{2}}+Y^{\frac{p_{2}}{2}}+Z^{p_{3}}\end{array}\right\}$ \\
\hline IIA & $x^{p_{2}}+x y^{\frac{p_{3}}{p_{2}}}+z^{p_{1}}-2 x^{\frac{p_{2}+1}{2}} y^{\frac{p_{3}}{2 p_{2}}}$ & $\left\{\begin{array}{c}X Y-W^{2} \\
X W+Y^{\frac{p_{3}}{2 p_{2}}}+Z^{p_{1}}\end{array}\right\}$ \\
\hline IIB & $x^{p_{1}}+y^{p_{2}}+y z^{\frac{p_{3}}{p_{2}}}-2 x^{\frac{p_{1}}{2}} y^{\frac{p_{2}}{2}}$ & $\left\{\begin{array}{c}X Y-W^{2} \\
X^{\frac{p_{1}}{2}}+Y^{\frac{p_{2}}{2}} Z+Z^{\frac{p_{3}}{p_{2}}}\end{array}\right\}$ \\
\hline $\mathrm{IIB}^{\sharp}$ & $-x^{\frac{p_{1}}{2}} z^{\frac{p_{3}}{2}}+y^{2}+y z^{\frac{p_{3}}{2}}-x^{\frac{p_{1}}{2}} y$ & $\left\{\begin{array}{c}X Y-Z W \\
X^{\frac{p_{1}}{2}}+Y W+Z^{\frac{p_{3}}{2}}\end{array}\right\}$ \\
\hline III & $x^{q_{2}+1} y+x y^{q_{3}+1}+z^{p_{1}}-2 x^{\frac{q_{2}}{2}+1} y^{\frac{q_{3}}{2}+1}$ & $\left\{\begin{array}{c}X Y-W^{2} \\
\left(X^{\frac{q_{2}}{2}}+Y^{\frac{q_{3}}{2}}\right) W+Z^{p_{1}}\end{array}\right\}$ \\
\hline IV & $x^{p_{1}}+x y^{\frac{p_{2}}{p_{1}}}+y z^{\frac{p_{3}}{p_{2}}}-2 x^{\frac{p_{1}+1}{2}} y^{\frac{p_{2}}{2 p_{1}}}$ & $\left\{\begin{array}{c}X Y-W^{2} \\
X^{\frac{p_{1}-1}{2}} W+Y^{\frac{p_{2}}{2 p_{1}}} Z+Z^{\frac{p_{3}}{p_{2}}}\end{array}\right.$ \\
\hline IV $\sharp$ & $-x^{\frac{p_{1}-1}{2}} z^{\frac{p_{3}}{p_{2}}}+x y^{2}+y z^{\frac{p_{3}}{p_{2}}}-x^{\frac{p_{1}+1}{2}} y$ & $\left\{\begin{array}{c}X Y-Z W \\
X^{\frac{p_{1}-1}{2}} W+Y W+Z^{\frac{p_{3}}{p_{2}}}\end{array}\right\}$ \\
\hline
\end{tabular}

\section{Virtual Singularities}

We now associate other equations to the polynomials from above. For each type, consider the polynomial $\mathbf{f}$ from Table 1 and assume that the conditions indicated in Table 2 are satisfied. In all cases except $\operatorname{IIB}^{\sharp}$ and $\operatorname{IV}^{\sharp}$, the polynomial $\mathbf{f}(x, y, z)$ is of the form

$$
\mathbf{f}(x, y, z)=u(x, y, z)+v(x, y, z)\left(x-y^{e}\right)^{2}
$$

or

$$
\mathbf{f}(x, y, z)=u(x, y, z)+v(x, y, z)\left(y-x^{e}\right)^{2}
$$

for some monomials $u(x, y, z)$ and $v(x, y, z)$ and some integer $e \geq 2$. We consider the cusp singularity $\mathbf{f}(x, y, z)-x y z$ and perform the coordinate change $x \mapsto x+y^{e}$ or $y \mapsto y+x^{e}$ respectively. The corresponding coordinate change is indicated in Table 2. Then $\mathbf{f}(x, y, z)-x y z$ is transformed to $\mathbf{h}(x, y, z)-x y z$ where $\mathbf{h}(x, y, z)$ is indicated in the last column of Table 2.

By inspection of Table 2, we see that some of the polynomials $\mathbf{h}$ have 4 monomials and others only 3 . We restrict our consideration to the cases where the polynomial $\mathbf{h}$ has 4 monomials. These cases are listed in Table 3 . The singularities defined by the polynomials $\mathbf{h}(x, y, z)$ will be called virtual singularities. We consider the duality between the virtual singularities on one side and the complete intersection singularities on the other side. 
Table 2 Conditions and transformations

\begin{tabular}{llll}
\hline Type & Conditions & Coord. change & $\mathbf{h}(x, y, z)$ \\
\hline I & $p_{2}=2$ & $y \mapsto y+x^{\frac{p_{1}}{2}}$ & $-x^{\frac{p_{1}}{2}+1} z+y^{2}+z^{p_{3}}$ \\
IIA & $p_{2}=3$ & $x \mapsto x+y^{\frac{p_{3}}{6}}$ & $-y^{\frac{p_{3}}{6}+1} z+z^{p_{1}}+x^{3}+x^{2} y^{\frac{p_{3}}{6}}$ \\
IIA & $\frac{p_{3}}{p_{2}=2}$ & $y \mapsto y+x^{\frac{p_{2}-1}{2}}$ & $-x^{\frac{p_{2}+1}{2}} z+z^{p_{1}}+x y^{2}$ \\
IIB & $p_{1}=2$ & $x \mapsto x+y^{\frac{p_{2}}{2}}$ & $-y^{\frac{p_{2}}{2}+1} z+x^{2}+y z^{\frac{p_{3}}{p 2}}$ \\
IIB & $p_{2}=2$ & $y \mapsto y+x^{\frac{p_{1}}{2}}$ & $-x^{\frac{p_{1}}{2}+1} z+y^{2}+y z^{\frac{p_{3}}{2}}+x^{\frac{p_{1}}{2}} z^{\frac{p_{3}}{2}}$ \\
IIB $\#$ & $p_{2}=2$ & $y \mapsto y+x^{\frac{p_{1}}{2}}$ & $-x^{\frac{p_{1}}{2}+1} z+y^{2}+y z^{\frac{p_{3}}{2}}+x^{\frac{p_{1}}{2}} y$ \\
III & $q_{2}=2$ & $x \mapsto x+y^{\frac{q_{3}}{2}}$ & $-y^{\frac{q_{3}}{2}+1} z+z^{p_{1}}+x^{3} y+x^{2} y^{\frac{q_{3}}{2}+1}$ \\
IV & $p_{1}=3$ & $x \mapsto x+y^{\frac{p_{2}}{6}}$ & $-y^{\frac{p_{2}}{6}+1} z+x^{3}+y z^{\frac{p_{3}}{p_{2}}}+x^{2} y^{\frac{p_{2}}{6}}$ \\
IV $_{2}$ & $\frac{p_{2}}{p_{1}}=2$ & $y \mapsto y+x^{\frac{p_{1}-1}{2}}$ & $-x^{\frac{p_{1}+1}{2}} z+x y^{2}+y z^{\frac{p_{2}}{p_{2}}}+x^{\frac{p_{1}-1}{2}} z^{\frac{p_{3}}{p 2}}$ \\
$\mathrm{IV}_{2}^{\sharp}$ & $\frac{p_{2}}{p_{1}}=2$ & $y \mapsto y+x^{\frac{p_{1}-1}{2}}$ & $-x^{\frac{p_{1}+1}{2}} z+x y^{2}+y z^{\frac{p_{3}}{p_{2}}}+x^{\frac{p_{1}+1}{2}} y$ \\
\hline
\end{tabular}

Let

$$
\mathbf{h}(x, y, z)=\sum_{i=1}^{4} a_{i} x^{A_{i 1}} y^{A_{i 2}} z^{A_{i 3}}
$$

be the polynomial defining a virtual singularity and let $\operatorname{Supp}(\mathbf{h})=\left\{\left(A_{i 1}, A_{i 2}, A_{i 3}\right) \in\right.$ $\left.\mathbb{Z}^{3} \mid i=1, \ldots, 4\right\}$. Let $\Delta_{\infty}(\mathbf{h})$ be the Newton polygon of $\mathbf{h}$ at infinity (Kouchnirenko 1976), i.e. $\Delta_{\infty}(\mathbf{h})$ is the convex closure in $\mathbb{R}^{n}$ of $\operatorname{Supp}(\mathbf{h}) \cup\{0\}$. The Newton polygon $\Delta_{\infty}(\mathbf{h})$ has two faces which do not contain the origin. Call these faces $\Sigma_{1}$ and $\Sigma_{2}$. Let $I_{k}:=\left\{i \in\{1, \ldots, 4\} \mid\left(A_{i 1}, A_{i 2}, A_{i 3}\right) \in \Sigma_{k}\right\}, k=1,2$, and let

$$
\mathbf{h}_{k}=\sum_{i \in I_{k}} a_{i} x^{A_{i 1}} y^{A_{i 2}} z^{A_{i 3}} .
$$

Then $\mathbf{h}_{k}$ is an invertible polynomial with a non-isolated singularity at the origin. The polynomials $\mathbf{h}_{1}$ and $\mathbf{h}_{2}$ are listed in Table 3 . Their canonical systems of weights are reduced. One of the systems of weights of $\mathbf{h}_{1}$ and $\mathbf{h}_{2}$ coincides with the reduced system of weights of the non-degenerate invertible polynomial $f$ we started with. Let the numbering be chosen such that this is the system of weights of $\mathbf{h}_{2}$. The systems of weights are listed in Table 4.

The dual complete intersection singularity defined by $\widetilde{\mathbf{f}}_{1}=\widetilde{\mathbf{f}}_{2}=0$ is weighted homogeneous. We list the systems of weights of these complete intersection singularities in Table 5. It turns out that the degrees of the systems of weights of $\mathbf{h}_{1}$ and $\mathbf{h}_{2}$ coincide with the degrees of the two polynomials $\widetilde{\mathbf{f}}_{1}$ and $\widetilde{\mathbf{f}}_{2}$ respectively. 
Table 3 Virtual singularities

\begin{tabular}{llll}
\hline Type & & $\mathbf{h}_{1}(x, y, z)$ & $\mathbf{h}_{2}(x, y, z)$ \\
\hline IIA & $p_{2}=3$ & $-y^{\frac{p_{3}}{6}+1} z+z^{p_{1}}+x^{2} y^{\frac{p_{3}}{6}}$ & $z^{p_{1}}+x^{3}+x^{2} y^{\frac{p_{3}}{6}}$ \\
IIB & $p_{2}=2$ & $-x^{\frac{p_{1}}{2}+1} z+y^{2}+x^{\frac{p_{1}}{2}} z^{\frac{p_{3}}{2}}$ & $y^{2}+y z^{\frac{p_{3}}{2}}+x^{\frac{p_{1}}{2}} z^{\frac{p_{3}}{2}}$ \\
IIB & $p_{2}=2$ & $-x^{\frac{p_{1}}{2}+1} z+y z^{\frac{p_{3}}{2}}+x^{\frac{p_{1}}{2}} y$ & $y^{2}+y z^{\frac{p_{3}}{2}}+x^{\frac{p_{1}}{2}} y$ \\
III & $q_{2}=2$ & $-y^{\frac{q_{3}}{2}+1} z+z^{p_{1}}+x^{2} y^{\frac{q_{3}}{2}+1}$ & $z^{p_{1}}+x^{3} y+x^{2} y^{\frac{q_{3}}{2}+1}$ \\
IV $_{1}$ & $p_{1}=3$ & $-y^{\frac{p_{2}}{6}+1} z+y z^{\frac{p_{3}}{p_{2}}}+x^{2} y^{\frac{p_{2}}{6}}$ & $x^{3}+y z^{\frac{p_{3}}{p_{2}}}+x^{2} y^{\frac{p_{2}}{6}}$ \\
$\mathrm{IV}_{2}$ & $\frac{p_{2}}{p_{1}}=2$ & $-x^{\frac{p_{1}+1}{2}} z+x y^{2}+x^{\frac{p_{1}-1}{2}} z^{\frac{p_{3}}{p_{2}}}$ & $x y^{2}+y z^{\frac{p_{3}}{p_{2}}}+x^{\frac{p_{1}-1}{2}} z^{\frac{p_{3}}{p_{2}}}$ \\
$\mathrm{IV}_{2}^{\#}$ & $\frac{p_{2}}{p_{1}}=2$ & $-x^{\frac{p_{1}+1}{2}} z+y z^{\frac{p_{3}}{p_{2}}}+x^{\frac{p_{1}+1}{2}} y$ & $x y^{2}+y z^{\frac{p_{3}}{p_{2}}}+x^{\frac{p_{1}+1}{2}} y$ \\
\hline
\end{tabular}

Table 4 Systems of weights corresponding to the virtual singularities

\begin{tabular}{lll}
\hline Type & System of weights of $\mathbf{h}_{1}$ & System of weights of $\mathbf{h}_{2}$ \\
\hline IIA & $\left(p_{1}+\frac{p_{3}}{6}, 2 p_{1}-2, \frac{p_{3}}{3}+2 ; p_{1}\left(\frac{p_{3}}{3}+2\right)\right)$ & $\left(\frac{p_{1} p_{3}}{6}, p_{1}, \frac{p_{3}}{2} ; \frac{p_{1} p_{3}}{2}\right)$ \\
IIB & $\left(p_{3}-2, \frac{p_{1} p_{3}}{4}+\frac{p_{3}}{2}-\frac{p_{1}}{2}, 2 ; \frac{p_{1} p_{3}}{2}+p_{3}-p_{1}\right)$ & $\left(\frac{p_{3}}{2}, \frac{p_{1} p_{3}}{4}, \frac{p_{1}}{2} ; \frac{p_{1} p_{3}}{2}\right)$ \\
IIB $^{\sharp}$ & $\left(\frac{p_{3}}{2}, \frac{p_{1}}{2}+\frac{p_{3}}{2}, \frac{p_{1}}{2} ; \frac{p_{1} p_{3}}{4}+\frac{p_{3}}{2}+\frac{p_{1}}{2}\right)$ & $\left(\frac{p_{3}}{2}, \frac{p_{1} p_{3}}{4}, \frac{p_{1}}{2} ; \frac{p_{1} p_{3}}{2}\right)$ \\
III & $\left(\frac{q_{3}}{2}+1,2 p_{1}-2, q_{3}+2 ; p_{1}\left(q_{3}+2\right)\right)$ & $\left(\frac{q_{3}}{2} p_{1}, p_{1}, 3 \frac{q_{3}}{2}+1 ; p_{1}\left(3 \frac{q_{3}}{2}+1\right)\right)$ \\
IV $_{1}$ & $\left(\frac{p_{2}}{6}+\frac{p_{3}}{p_{2}}-1,2 \frac{p_{3}}{p_{2}}-2, \frac{p_{2}}{3} ; \frac{p_{3}}{3}+2 \frac{p_{3}}{p_{2}}-2\right)$ & $\left(\frac{p_{3}}{6}, \frac{p_{3}}{p_{2}}, \frac{p_{2}}{2}-1 ; \frac{p_{3}}{2}\right)$ \\
IV $_{2}$ & $\left(2 \frac{p_{3}}{p_{2}}-2, \frac{p_{3}}{4}-\frac{p_{3}}{2 p_{2}}-\frac{p_{1}}{2}+\frac{3}{2}, 2 ; \frac{p_{3}}{2}+\frac{p_{3}}{p_{2}}-p_{1}+1\right)$ & $\left(\frac{p_{3}}{p_{2}},\left(p_{1}-1\right) \frac{p_{3}}{2 p_{2}}, \frac{p_{1}+1}{2} ; \frac{p_{3}}{2}\right)$ \\
IV $_{2}^{\sharp}$ & $\left(\frac{p_{3}}{p_{2}}, \frac{p_{1}+1}{2}, \frac{p_{1}+1}{2} ; \frac{p_{3}}{4}+\frac{p_{3}}{2 p_{2}}+\frac{p_{1}}{2}+\frac{1}{2}\right)$ & $\left(\frac{p_{3}}{p_{2}},\left(p_{1}-1\right) \frac{p_{3}}{2 p_{2}}, \frac{p_{1}+1}{2} ; \frac{p_{3}}{2}\right)$ \\
\hline
\end{tabular}

Table 5 Systems of weights of the pairs $\left(\widetilde{\mathbf{f}}_{1}, \widetilde{\mathbf{f}}_{2}\right)$

\begin{tabular}{ll}
\hline Type & System of weights \\
\hline $\mathrm{IIA}$ & $\left(p_{1}\left(\frac{p_{3}}{3}-1\right), 3 p_{1}, \frac{p_{3}}{2}, \frac{p_{1}}{2}\left(\frac{p_{3}}{3}+2\right) ; p_{1}\left(\frac{p_{3}}{3}+2\right), \frac{p_{1} p_{3}}{2}\right)$ \\
$\mathrm{IIB}$ & $\left(p_{3}, \frac{p_{1} p_{3}}{2}-p_{1}, p_{1}, \frac{p_{1} p_{3}}{4}+\frac{p_{3}}{2}-\frac{p_{1}}{2} ; \frac{p_{1} p_{3}}{2}+p_{3}-p_{1}, \frac{p_{1} p_{3}}{2}\right)$ \\
$\mathrm{IIB}^{\sharp}$ & $\left(p_{3}, \frac{p_{1} p_{3}}{4}+\frac{p_{1}}{2}-\frac{p_{3}}{2}, p_{1}, \frac{p_{1} p_{3}}{4}+\frac{p_{3}}{2}-\frac{p_{1}}{2} ; \frac{p_{1} p_{3}}{4}+\frac{p_{3}}{2}+\frac{p_{1}}{2}, \frac{p_{1} p_{3}}{2}\right)$ \\
$\mathrm{III}$ & $\left(p_{1} q_{3}, 2 p_{1}, 3 \frac{q_{3}}{2}+1,\left(\frac{q_{3}}{2}+1\right) p_{1} ; p_{1}\left(q_{3}+2\right), p_{1}\left(3 \frac{q_{3}}{2}+1\right)\right)$ \\
$\mathrm{IV}_{1}$ & $\left(\frac{p_{3}}{3}-\frac{p_{3}}{p_{2}}+1,3\left(\frac{p_{3}}{p_{2}}-1\right), \frac{p_{2}}{2}, \frac{p_{3}}{6}+\frac{p_{3}}{p_{2}}-1 ; \frac{p_{3}}{3}+2 \frac{p_{3}}{p_{2}}-2, \frac{p_{3}}{2}\right)$ \\
$\mathrm{IV}_{2}$ & $\left(\frac{p_{3}}{p_{2}}+1, \frac{p_{3}}{2}-p_{1}, p_{1}, \frac{p_{3}}{4}+\frac{p_{3}}{2 p_{2}}-\frac{p_{1}}{2}+\frac{1}{2} ; \frac{p_{3}}{2}+\frac{p_{3}}{p_{2}}-p_{1}+1, \frac{p_{3}}{2}\right)$ \\
$\mathrm{IV}_{2}^{\sharp}$ & $\left(\frac{p_{3}}{p_{2}}+1, \frac{p_{3}}{4}-\frac{p_{3}}{2 p_{2}}+\frac{p_{1}}{2}-\frac{1}{2}, p_{1}, \frac{p_{3}}{4}+\frac{p_{3}}{2 p_{2}}-\frac{p_{1}}{2}+\frac{1}{2} ; \frac{p_{3}}{4}+\frac{p_{3}}{2 p_{2}}+\frac{p_{1}}{2}+\frac{1}{2}, \frac{p_{3}}{2}\right)$ \\
\hline
\end{tabular}

\section{Dolgachev and Gabrielov Numbers}

We shall now define Dolgachev and Gabrielov numbers for the polynomials $\mathbf{h}$ and the pairs of polynomials $\left(\widetilde{\mathbf{f}}_{1}, \widetilde{\mathbf{f}}_{2}\right)$ occurring in our duality. 
We first define these numbers for the pairs $\left(\widetilde{\mathbf{f}}_{1}, \widetilde{\mathbf{f}}_{2}\right)$. Let $X_{\widetilde{\mathbf{f}}_{1}, \widetilde{\mathbf{f}}_{2}} \subset \mathbb{C}^{4}$ be the weighted homogeneous complete intersection in $\mathbb{C}^{4}$ defined by the two equations $\widetilde{\mathbf{f}}_{1}(W, X, Y, Z)=\widetilde{\mathbf{f}}_{2}(W, X, Y, Z)=0$, where $\widetilde{\mathbf{f}}_{1}(W, X, Y, Z)=X Y-W^{2}$ or $\widetilde{\mathbf{f}}_{1}(W, X, Y, Z)=X Y-Z W$.

Definition Let $\mathcal{C}_{\widetilde{\mathbf{f}}_{1}}, \widetilde{\mathbf{f}}_{2}:=\left[\left(X_{\widetilde{\mathbf{f}}_{1}, \widetilde{\mathbf{f}}_{2}} \backslash\{0\}\right) / \mathbb{C}^{*}\right]$. Then $\mathcal{C}_{\widetilde{\mathbf{f}}_{1}, \widetilde{\mathbf{f}}_{2}}$ is a smooth projective curve with three isotropic points of orders $\alpha_{1}, \alpha_{2}, \alpha_{3}$. We call these numbers the Dolgachev numbers of the pair $\left(\widetilde{\mathbf{f}}_{1}, \widetilde{\mathbf{f}}_{2}\right)$.

The Gabrielov numbers are defined similarly as in the hypersurface case. We consider the complete intersection singularity $\left(X^{\prime}, 0\right)$ defined by

$$
\left\{\begin{array}{l}
\widetilde{\mathbf{f}}_{1}(W, X, Y, Z), \\
\widetilde{\mathbf{f}}_{2}(W, X, Y, Z)-Z W .
\end{array}\right.
$$

As in Ebeling and Takahashi (2011) one can show that one can find a holomorphic change of coordinates such that the singularity $\left(X^{\prime}, 0\right)$ is also given by equations of the form

$$
\left\{\begin{array}{l}
X Y-Z^{\gamma_{1}}-W^{\gamma_{2}} \\
X^{\gamma_{3}}+Y^{\gamma_{4}}-Z W
\end{array}\right.
$$

This means that $\left(X^{\prime}, 0\right)$ is a cusp singularity of type $T_{\gamma_{1}, \gamma_{3}, \gamma_{2}, \gamma_{4}}^{2}$ in the notation of Ebeling (1987, 3.1).

Definition The Gabrielov numbers of the pair $\left(\widetilde{\mathbf{f}}_{1}, \widetilde{\mathbf{f}}_{2}\right)$ are the numbers $\left(\gamma_{1}, \gamma_{2} ; \gamma_{3}, \gamma_{4}\right)$.

The Dolgachev and Gabrielov numbers for the pairs $\left(\widetilde{\mathbf{f}}_{1}, \widetilde{\mathbf{f}}_{2}\right)$ of Table 1 are indicated in Table 6.

Now let $\mathbf{h}$ be the polynomial of Table 3 defining a virtual singularity. The Gabrielov numbers of $\mathbf{h}$ are defined as in Ebeling and Takahashi (2011). Namely, we consider

Table 6 Pairs $\left(\widetilde{\mathbf{f}}_{1}, \widetilde{\mathbf{f}}_{2}\right)$ : Dolgachev and Gabrielov numbers

\begin{tabular}{lll}
\hline Type & Dolgachev & Gabrielov \\
\hline I & $p_{2}, p_{3}, p_{1}$ & $2,2 p_{3}-2 ; \frac{p_{1}}{2}, \frac{p_{2}}{2}$ \\
IIA & $p_{1}, p_{2},\left(\frac{p_{3}}{p_{2}}-1\right) p_{1}$ & $2,2 p_{1}-2 ; \frac{p_{1}\left(p_{2}-1\right)}{2}, \frac{p_{3}}{2 p_{2}}$ \\
IIB & $p_{1}, p_{2},\left(\frac{p_{3}}{p_{2}}-1\right) p_{1}$ & $2,2 \frac{p_{3}}{p_{2}}-2 ; \frac{p_{1}}{2}, \frac{p_{1}\left(p_{2}-1\right)}{2}$ \\
IIB $\sharp$ & $\frac{p_{3}}{2}\left(\frac{p_{1}}{2}-1\right)+\frac{p_{1}}{2}, 2, \frac{p_{1}}{2}\left(\frac{p_{3}}{2}-1\right)+\frac{p_{3}}{2}$ & $\frac{p_{3}}{2}, \frac{p_{1}}{2} ; \frac{p_{3}}{2}, \frac{p_{1}}{2}$ \\
III & $p_{1} q_{2}, p_{1} q_{3}, p_{1}$ & $2,2 p_{1}-2 ; p_{1}, \frac{q_{3}}{2} p_{1}$ \\
IV & $p_{1}, \frac{p_{3}}{p_{1}}-\frac{p_{3}}{p_{2}}+1,\left(\frac{p_{3}}{p_{2}}-1\right) p_{1}$ & $2,2 \frac{p_{3}}{p_{2}}-2 ; \frac{1}{2} \frac{p_{3}}{p_{2}}\left(p_{1}-1\right), \frac{1}{2}\left(p_{2}-p_{1}+1\right)$ \\
IV $\sharp$ & $\frac{p_{1}-1}{2}\left(\frac{p_{3}}{p_{2}}+1\right), \frac{p_{3}}{p_{2}}+1, \frac{p_{1}+1}{2}\left(\frac{p_{3}}{p_{2}}-1\right)+1$ & $\frac{p_{3}}{p_{2}}, \frac{1}{2}\left(p_{1}+1\right) ; \frac{p_{3}}{p_{2}}, \frac{1}{2} \frac{p_{3}}{p_{2}}\left(p_{1}-1\right)$ \\
\hline
\end{tabular}


the polynomial $\mathbf{h}(x, y, z)-x y z$. As in Ebeling and Takahashi (2011), one can show that the germ at the origin of this polynomial is right equivalent to a cusp singularity

$$
x^{\gamma_{1}}+y^{\gamma_{2}}+z^{\gamma_{3}}-x y z
$$

i.e. it can be transformed to such a polynomial by a holomorphic change of coordinates at the origin. We define the Gabrielov numbers of $\mathbf{h}$ to be the triple $\left(\gamma_{1}, \gamma_{2}, \gamma_{3}\right)$.

Example 3 We illustrate how to find the corresponding holomorphic coordinate change by two examples.

(a) IIA $\left(p_{2}=3\right)$ : Here $\mathbf{h}(x, y, z)=-y^{\frac{p_{3}}{6}+1} z+z^{p_{1}}+x^{3}+x^{2} y^{\frac{p_{3}}{6}}$. The substitution $x \mapsto x-y^{\frac{p_{3}}{6}}$ transforms the polynomial $\mathbf{h}(x, y, z)-x y z$ back to

$$
\mathbf{f}(x, y, z)-x y z=x^{3}+x y^{\frac{p_{3}}{3}}+z^{p_{1}}-2 x^{2} y^{\frac{p_{3}}{6}}-x y z .
$$

The substitution $z \mapsto z+y^{\frac{p_{3}}{3}-1}$ transforms this polynomial to

$$
\begin{aligned}
& x^{3}+x y^{\frac{p_{3}}{3}}+\left(z+y^{\frac{p_{3}}{3}-1}\right)^{p_{1}}-2 x^{2} y^{\frac{p_{3}}{6}}-x y^{\frac{p_{3}}{3}}-x y z \\
& =x^{3}+y^{\left(\frac{p_{3}}{3}-1\right) p_{1}}+z^{p_{1}}-x y z+\ldots
\end{aligned}
$$

where the monomial $x y^{\frac{p_{3}}{3}}$ is cancelled and the dots refer to other monomials involving more than one variable. According to Arnold (1974, Lemma 7.3), by similar transformations, one can get rid of the monomials of lowest degree involving more than one variable by possibly introducing new such monomials, but of higher degree. The pure powers of single variables of lowest degree are preserved. In this way, we see that we get the Gabrielov numbers in the first line of Table 7. See also Example 8 in Sect. 7 for a concrete example and more details on this type of deformation of the polynomial $\mathbf{h}(x, y, z)$.

(b) $\operatorname{IV}_{2}\left(\frac{p_{2}}{p_{1}}=2\right)$ : Here $\mathbf{h}(x, y, z)=-x^{\frac{p_{1}+1}{2}} z+x y^{2}+y z^{\frac{p_{3}}{p_{2}}}+x^{\frac{p_{1}-1}{2}} z^{\frac{p_{3}}{p_{2}}}$. Again we work with

$$
\mathbf{f}(x, y, z)-x y z=x^{p_{1}}+x y^{2}+y z^{\frac{p_{3}}{p_{2}}}-2 x^{\frac{p_{1}+1}{2}} y-x y z .
$$

The substitution $z \mapsto z+y$ followed by $x \mapsto x+z^{\frac{p_{3}}{p_{2}}-1}$ transforms this polynomial to

$$
x^{p_{1}}+y^{\frac{p_{3}}{p_{2}}+1}+z^{\left(\frac{p_{3}}{p_{2}}-1\right) p_{1}}-x y z+\ldots
$$

where the dots again refer to certain mixed terms. By arguments as above, we can get rid of the mixed terms. This yields the Gabrielov numbers in Table 7. (Note that $\frac{p_{2}}{p_{1}}=2$ implies that $\frac{p_{3}}{p_{2}}+1=\frac{p_{3}}{p_{1}}-\frac{p_{3}}{p_{2}}+1$.)

The Dolgachev numbers of the polynomial $\mathbf{h}$ are defined as follows. We associated to $\mathbf{h}$ two weighted homogeneous polynomials $\mathbf{h}_{1}$ and $\mathbf{h}_{2}$. Let $i=1,2$ and let $V_{i}:=$ 
Table 7 Virtual singularities: Dolgachev and Gabrielov numbers

\begin{tabular}{lll}
\hline Type & Dolgachev & Gabrielov \\
\hline IIA & $2,2 p_{1}-2 ; p_{1}, \frac{p_{3}}{6}$ & $3,\left(\frac{p_{3}}{3}-1\right) p_{1}, p_{1}$ \\
IIB & $2,2 \frac{p_{3}}{p_{2}}-2 ; \frac{p_{1}}{2}, \frac{p_{1}}{2}$ & $p_{1}, 2,\left(\frac{p_{3}}{2}-1\right) p_{1}$ \\
IIB $^{\sharp}$ & $\frac{p_{3}}{2}, \frac{p_{1}}{2} ; \frac{p_{3}}{2}, \frac{p_{1}}{2}$ & $\frac{p_{3}}{2}\left(\frac{p_{1}}{2}-1\right)+\frac{p_{1}}{2}, 2, \frac{p_{1}}{2}\left(\frac{p_{3}}{2}-1\right)+\frac{p_{3}}{2}$ \\
III & $2,2 p_{1}-2 ; p_{1}, \frac{q_{3}}{2} p_{1}$ & $2 p_{1}, q_{3} p_{1}, p_{1}$ \\
IV $_{1}$ & $2,2 \frac{p_{3}}{p_{2}}-2 ; \frac{p_{3}}{p_{2}}, \frac{1}{2}\left(p_{2}-2\right)$ & $3, \frac{p_{3}}{3}-\frac{p_{3}}{p_{2}}+1,3\left(\frac{p_{3}}{p_{2}}-1\right)$ \\
IV $_{2}$ & $2,2 \frac{p_{3}}{p_{2}}-2 ; \frac{1}{2} \frac{p_{3}}{p_{2}}\left(p_{1}-1\right), \frac{1}{2}\left(p_{1}+1\right)$ & $p_{1}, \frac{p_{3}}{p_{1}}-\frac{p_{3}}{p_{2}}+1,\left(\frac{p_{3}}{p_{2}}-1\right) p_{1}$ \\
$\mathrm{IV}_{2}^{\sharp}$ & $\frac{p_{3}}{p_{2}}, \frac{1}{2}\left(p_{1}+1\right) ; \frac{p_{3}}{p_{2}}, \frac{1}{2} \frac{p_{3}}{p_{2}}\left(p_{1}-1\right)$ & $\frac{p_{1}-1}{2}\left(\frac{p_{3}}{p_{2}}+1\right), \frac{p_{3}}{p_{2}}+1, \frac{p_{1}+1}{2}\left(\frac{p_{3}}{p_{2}}-1\right)+1$ \\
\hline
\end{tabular}

$\left\{(x, y, z) \in \mathbb{C}^{3} \mid \mathbf{h}_{i}(x, y, z)=0\right\}$. We consider the $\mathbb{C}^{*}$-action on $V_{i}$ given by the system of weights of $\mathbf{h}_{i}$ (see Table 4). We consider the exceptional orbits (i.e. orbits with a non-trivial isotropy group) of this action. We distinguish between two cases:

(A) $V_{i}$ contains a coordinate hyperplane.

(B) $V_{i}$ does not contain a coordinate hyperplane.

In case (A) we consider those exceptional orbits which are not contained in the coordinate hyperplane which is contained in $V_{i}$. In case (B) we consider those exceptional orbits which do not coincide with the singular locus of $V_{i}$. We call these the principal orbits. It turns out that in all cases we have exactly two principal orbits.

Example 4 (a) IIA $\left(p_{2}=3\right): \mathbf{h}_{1}(x, y, z)=-y^{\frac{p_{3}}{6}+1} z+z^{p_{1}}+x^{2} y^{\frac{p_{3}}{6}}$ with the system of weights $\left(p_{1}+\frac{p_{3}}{6}, 2 p_{1}-2, \frac{p_{3}}{3}+2 ; p_{1}\left(\frac{p_{3}}{3}+2\right)\right)$. The exceptional orbits are:

$$
\begin{aligned}
& y=z=0 \text { singular line } \\
& x=-y^{\frac{p_{3}}{6}+1} z+z^{p_{1}}=0 \text { order of isotropy group : } 2 \\
& x=z=0 \text { order of isotropy group: } 2 p_{1}-2
\end{aligned}
$$

(b) $\operatorname{IV}_{2}\left(\frac{p_{2}}{p_{1}}=2\right): \mathbf{h}_{1}(x, y, z)=-x^{\frac{p_{1}+1}{2}} z+x y^{2}+x^{\frac{p_{1}-1}{2}} z^{\frac{p_{3}}{p_{2}}}=x\left(-x^{\frac{p_{1}-1}{2}} z+y^{2}+\right.$ $\left.x^{\frac{p_{1}-3}{2}} z^{\frac{p_{3}}{p_{2}}}\right)$ with the system of weights $\left(2 \frac{p_{3}}{p_{2}}-2, \frac{p_{3}}{4}-\frac{p_{3}}{2 p_{2}}-\frac{p_{1}}{2}+\frac{3}{2}, 2 ; \frac{p_{3}}{2}+\frac{p_{3}}{p_{2}}-p_{1}+1\right)$. The exceptional orbits not contained in the hyperplane $x=0$ are:

$$
\begin{aligned}
& y=z=0 \text { order of isotropy group: } 2 \frac{p_{3}}{p_{2}}-2 \\
& y=-x^{\frac{p_{1}+1}{2}} z+x^{\frac{p_{1}-1}{2}} z^{\frac{p_{3}}{p_{2}}}=0 \text { order of isotropy group: } 2
\end{aligned}
$$

Definition The Dolgachev numbers of $\mathbf{h}$ are the numbers $\alpha_{1}, \alpha_{2} ; \alpha_{3}, \alpha_{4}$ where $\alpha_{1}, \alpha_{2}$ and $\alpha_{3}, \alpha_{4}$ are the orders of the isotropy groups of the principal exceptional orbits of $\mathbf{h}_{1}$ and $\mathbf{h}_{2}$ respectively.

We list the Dolgachev and Gabrielov numbers of the polynomials $\mathbf{h}$ corresponding to the virtual singularities in Table 7. 


\section{Strange Duality}

Comparing Table 7 with Table 6, we obtain the following result.

Theorem 5 The Gabrielov numbers of the polynomial $\mathbf{h}$ corresponding to a virtual singularity coincide with the Dolgachev numbers of the dual pair $\left(\widetilde{\mathbf{f}}_{1}, \widetilde{\mathbf{f}}_{2}\right)$ and, vice versa, the Gabrielov numbers of a pair $\left(\widetilde{\mathbf{f}}_{1}, \widetilde{\mathbf{f}}_{2}\right)$ coincide with the Dolgachev numbers of the dual polynomial $\mathbf{h}$.

Let $f_{1}, \ldots, f_{k}$ be quasihomogeneous functions on $\mathbb{C}^{n}$ of degrees $d_{1}, \ldots, d_{k}$ with respect to weights $w_{1}, \ldots, w_{n}$. Here $w_{1}, \ldots, w_{n}$ are positive integers with $\operatorname{gcd}\left(w_{1}, \ldots, w_{n}\right)=1, f_{j}\left(\lambda^{w_{1}} x_{1}, \ldots, \lambda^{w_{n}} x_{n}\right)=\lambda^{d_{j}} f_{j}\left(x_{1}, \ldots, x_{n}\right), \lambda \in \mathbb{C}$. We suppose that the equations $f_{1}=f_{2}=\ldots=f_{k}=0$ define a complete intersection $X$ in $\mathbb{C}^{n}$. There is a natural $\mathbb{C}^{*}$-action on the space $\mathbb{C}^{n}$ defined by $\lambda *\left(x_{1}, \ldots, x_{n}\right)=$ $\left(\lambda^{w_{1}} x_{1}, \ldots, \lambda^{w_{n}} x_{n}\right), \lambda \in \mathbb{C}^{*}$.

Let $A=\mathbb{C}[x] /\left(f_{1}, \ldots, f_{k}\right)$ be the coordinate ring of $X$. There is a natural grading on the ring $A: A_{s}$ is the set of functions $g \in A$ such that $g(\lambda * x)=\lambda^{s} g(x)$. Let $P_{X}(t)=\sum_{s=0}^{\infty} \operatorname{dim} A_{s} \cdot t^{s}$ be the Poincaré series of the graded algebra $A=\oplus_{s=0}^{\infty} A_{s}$. One has

$$
P_{X}(t)=\frac{\prod_{j=1}^{k}\left(1-t^{d_{j}}\right)}{\prod_{i=1}^{n}\left(1-t^{w_{i}}\right)} .
$$

For $0 \leq j \leq k$, let $X^{(j)}$ be the complete intersection given by the equations $f_{1}=\cdots=$ $f_{j}=0\left(X^{(0)}=\mathbb{C}^{n}, X^{(k)}=X\right)$. The restriction of the function $f_{j}(j=1, \ldots, k)$ to the variety $X^{(j-1)}$ defines a locally trivial fibration $X^{(j-1)} \backslash X^{(j)} \rightarrow \mathbb{C}^{*}$. Let $V^{(j)}=$ $f_{j}^{-1}(1) \cap X^{(j-1)}$ be the (Milnor) fibre of this fibration (the fibre $V^{(j)}$ is not necessarily smooth) and $\varphi^{(j)}: V^{(j)} \rightarrow V^{(j)}$ be the classical monodromy transformation of it. For a map $\varphi: Z \rightarrow Z$ of a topological space $Z$, let $\zeta_{\varphi}(t)$ be its zeta function

$$
\zeta_{\varphi}(t)=\prod_{p \geq 0}\left\{\operatorname{det}\left(\mathrm{id}-\left.t \cdot \varphi_{*}\right|_{H_{p}(Z ; \mathbb{C})}\right)\right\}^{(-1)^{p}} .
$$

If, in the definition, we use the actions of the operators $\varphi_{*}$ on the homology groups $\bar{H}_{p}(Z ; \mathbb{Z})$ reduced modulo a point, we get the reduced zeta function

$$
\bar{\zeta}_{\varphi}(t)=\frac{\zeta_{\varphi}(t)}{(1-t)}
$$

Let

$$
\bar{\zeta}_{X, j}(t):=\bar{\zeta}_{\varphi^{(j)}}(t)
$$

If both $X^{(j)}$ and $X^{(j-1)}$ have isolated singularities at the origin then $\bar{H}_{p}\left(V^{(j)} ; \mathbb{Z}\right)$ is non-trivial only for $p=n-j$ and therefore, if $n-j \geq 1$,

$$
\left(\bar{\zeta}_{X, j}(t)\right)^{(-1)^{n-j}}=\operatorname{det}\left(\mathrm{id}-\left.t \cdot \varphi_{*}^{(j)}\right|_{H_{n-j}\left(V^{(j)} ; \mathbb{C}\right)}\right)
$$


is the characteristic polynomial of the classical monodromy operator $\varphi_{*}^{(j)}$.

One can show that $\left(\varphi_{*}^{(j)}\right)^{d_{j}}=\mathrm{id}$ and therefore $\bar{\zeta}_{X, j}(t)$ can be written in the form

$$
\prod_{\ell \mid d_{j}}\left(1-t^{\ell}\right)^{\alpha_{\ell}}, \quad \alpha_{\ell} \in \mathbb{Z}
$$

Following Saito $(1998 \mathrm{a}, \mathrm{b})$, we define the Saito dual to $\bar{\zeta}_{X, j}(t)$ to be the rational function

$$
\bar{\zeta}_{X, j}^{*}(t)=\prod_{m \mid d_{j}}\left(1-t^{m}\right)^{-\alpha_{\left(d_{j} / m\right)}}
$$

(note that different degrees $d_{j}$ are used for different $j$ ).

Let $Y^{(k)}=\left(X^{(k)} \backslash\{0\}\right) / \mathbb{C}^{*}$ be the space of orbits of the $\mathbb{C}^{*}$-action on $X^{(k)} \backslash\{0\}$ and $Y_{m}^{(k)}$ be the set of orbits for which the isotropy group is the cyclic group of order $m$. Let

$$
\operatorname{Or}_{X}(t):=\prod_{m \geq 1}\left(1-t^{m}\right)^{\chi\left(Y_{m}^{(k)}\right)}
$$

be the product of cyclotomic polynomials with exponents corresponding to the partition of the complete intersection $X=X^{(k)}$ into parts of different orbit types; here $\chi(Z)$ denotes the Euler characteristic of a topological space $Z$.

Let $(X, 0)$ be the virtual hypersurface singularity defined by $\mathbf{h}=0$ and $(\widetilde{X}, 0)$ be the dual complete intersection singularity given by the equations $\widetilde{\mathbf{f}}_{1}=\widetilde{\mathbf{f}}_{2}=0$ according to Theorem 5. The function $\operatorname{Or}_{\tilde{X}}(t)$ is equal to the polynomial

$$
\operatorname{Or}_{\widetilde{X}}(t):=\prod_{k=1}^{3}\left(1-t^{\alpha_{k}}\right) \cdot(1-t)^{-1},
$$

where $\alpha_{1}, \alpha_{2}, \alpha_{3}$ are the Dolgachev numbers of the pair $\left(\widetilde{\mathbf{f}}_{1}, \widetilde{\mathbf{f}}_{2}\right)$, see Sect. 5 .

Finally, let $\zeta_{X}(t)$ be the zeta function of the monodromy at infinity of $\mathbf{h}$ and

$$
\bar{\zeta}_{X}(t)=\frac{\zeta_{X}(t)}{(1-t)}
$$

be the reduced zeta function of $\mathbf{h}$.

Theorem 6 Under the conditions of Table 3 we have

$$
\bar{\zeta}_{X}(t)=P_{\widetilde{X}}(t) \cdot \operatorname{Or}_{\widetilde{X}}(t)
$$

Proof The zeta function $\zeta_{X}(t)$ can be computed from the Newton polygon of $\mathbf{h}$ at infinity by Libgober and Sperber (1995). From Tables 4, 5, and 6 we can derive the formula.

From Ebeling and Gusein-Zade (2004) we get the following corollary: 
Corollary 7 Under the conditions of Table 3 we have

$$
\bar{\zeta}_{X}(t)=\bar{\zeta}_{\widetilde{X}, 1}^{*}(t) \cdot \bar{\zeta}_{\widetilde{X}, 2}^{*}(t)
$$

\section{Examples}

Let $f(x, y, z)$ be a weighted homogeneous polynomial with reduced system of weights $W=\left(w_{1}, w_{2}, w_{3} ; d\right)$. The Gorenstein parameter $a_{f}$ of $f$ is defined to be

$$
a_{f}:=d-w_{1}-w_{2}-w_{3}
$$

We now consider the classification of virtual singularities according to the Gorenstein parameter $a_{f}$ of the non-degenerate invertible polynomial $f$.

The classification of the non-degenerate invertible polynomials $f$ with $\left[G_{f}: G_{0}\right]=$ 2 and with $a_{f}<0$ can be extracted from Ebeling and Takahashi (2013, Table 3). From this we derive the classification of virtual singularities given in Table 8 .

One can also classify the non-degenerate invertible polynomials with $\left[G_{f}: G_{0}\right]=$ 2 with $a_{f}=0,1$. It turns out that there are no such polynomials with $a_{f}=0$. The virtual singularities corresponding to polynomials with $a_{f}=1$ are listed in Table 9.

I turns out that the virtual singularities with $a_{f}=1$ are exactly the virtual singularities corresponding to the bimodal series. According to Arnold's classification Arnold (1975), there are 8 series of bimodal hypersurface singularities. The virtual bimodal singularities are defined by setting $k=-1$ in the equations of these singularities. The names of Arnold are used in Table 9 and the equations for $k=-1$ are listed in Table 10. We compare them with our polynomials $\mathbf{h}$. We also indicate the names of the dual isolated complete intersection singularities according to the notation of Wall (1983). It turns out that these are exactly the singularities in the extension of Arnold's strange duality of Ebeling and Wall (1985).

We indicate the values of the Dolgachev and Gabrielov numbers of the polynomials h associated to the virtual bimodal singularites and the Dolgachev and Gabrielov numbers of the corresponding dual pairs of polynomials defining the isolated complete intersection singularities (ICIS) in Table 11.

Let $\mathbf{h}(x, y, z)=0$ be the equation for one of the virtual bimodal singularities. By inspection, one sees that the germ at the origin is an exceptional unimodal singularity. The corresponding singularity is indicated in Table 12. Moreover the global polynomial

Table 8 Gorenstein parameter $<0$ cases

\begin{tabular}{llllll}
\hline Type & $p_{1}, p_{2}, p_{3}$ & $\mathbf{h}$ & Name & Dolgachev & Gabrielov \\
\hline IIA & $2,3,6$ & $-y^{2} z+z^{2}+x^{3}+x^{2} y$ & $J_{1,-1}$ & 2,$2 ; 2,1$ & $2,3,2$ \\
IIB & $2,2,2 k$ & $-x^{2} z+y^{2}+y z^{k}+x z^{k}$ & $A_{2 k-1,-1}$ & $2,2 k-1 ; 1,1$ & $2,2,2 k-2$ \\
IIB $^{\sharp}$ & $2,2,2 k$ & $-x^{2} z+y^{2}+y z^{k}+x y$ & $A_{2 k-1,-1}^{\sharp}$ & $k, 1 ; k, 1$ & $1,2,2 k-1$ \\
\hline
\end{tabular}


Table 9 Gorenstein parameter 1 cases

\begin{tabular}{llllll}
\hline Type & $p_{1}, p_{2}\left(q_{2}\right), p_{3}\left(q_{3}\right)$ & $\mathbf{h}$ & Name & Dolgachev & Gabrielov \\
\hline IIA & $2,3,18$ & $-y^{4} z+z^{2}+x^{3}+x^{2} y^{3}$ & $J_{3,-1}$ & 2,$2 ; 2,3$ & $2,3,10$ \\
IIB & $4,2,6$ & $-x^{3} z+y^{2}+y z^{3}+x^{2} z^{3}$ & $Z_{1,-1}$ & 2,$4 ; 2,2$ & $4,2,8$ \\
IIB $^{\sharp}$ & $4,2,6$ & $-x^{3} z+y^{2}+y z^{3}+x^{2} y$ & $W_{1,-1}^{\sharp}$ & 3,$2 ; 3,2$ & $5,2,7$ \\
IIB & $6,2,4$ & $-x^{4} z+y^{2}+y z^{2}+x^{3} z^{2}$ & $W_{1,-1}$ & 2,$2 ; 3,3$ & $6,2,6$ \\
IIB & $6,2,4$ & $-x^{4} z+y^{2}+y z^{2}+x^{3} y$ & $W_{1,-1}^{\sharp}$ & 2,$3 ; 2,3$ & $7,2,5$ \\
III & $2,2,4$ & $-y^{3} z+z^{2}+x^{3} y+x^{2} y^{3}$ & $Z_{1,-1}$ & 2,$2 ; 2,4$ & $2,4,8$ \\
IV $_{2}$ & $3,6,18$ & $-x^{2} z+x y^{2}+y z^{3}+x z^{3}$ & $S_{1,-1}^{\sharp}$ & 2,$4 ; 3,2$ & $3,6,4$ \\
IV $_{2}^{\sharp}$ & $3,6,18$ & $-x^{2} z+x y^{2}+y z^{3}+x^{2} y$ & $U_{1,-1}$ & 3,$2 ; 3,3$ & $4,4,5$ \\
IV $_{1}$ & $3,12,24$ & $-y^{3} z+x^{3}+y z^{2}+x^{2} y^{2}$ & $Q_{2,-1}$ & 2,$2 ; 2,5$ & $3,3,7$ \\
IV $_{2}$ & $5,10,20$ & $-x^{3} z+x y^{2}+y z^{2}+x^{2} z^{2}$ & $S_{1,-1}$ & 2,$2 ; 4,3$ & $5,5,3$ \\
IV $_{2}^{\sharp}$ & $5,10,20$ & $-x^{3} z+x y^{2}+y z^{2}+x^{3} y$ & $S_{1,-1}^{\sharp}$ & 2,$3 ; 2,4$ & $6,3,4$ \\
\hline
\end{tabular}

Table 10 Bimodal virtual singularities

\begin{tabular}{lllll}
\hline Series & Arnold's equation & Type & $\mathbf{h}(x, y, z)$ & Dual \\
\hline$J_{3,-1}$ & $x^{3}+x^{2} y^{3}+z^{2}+y^{8}$ & IIA & $x^{3}+x^{2} y^{3}+z^{2}-y^{4} z$ & $J_{9}^{\prime}$ \\
$Z_{1,-1}$ & $x^{3} y+x^{2} y^{3}+z^{2}+y^{6}$ & III & $x^{3} y+x^{2} y^{3}+z^{2}-y^{3} z$ & $J_{10}^{\prime}$ \\
$Q_{2,-1}$ & $x^{3}+x^{2} y^{2}+y z^{2}+y^{5}$ & IV & $x^{3}+x^{2} y^{2}+y z^{2}-y^{3} z$ & $J_{11}^{\prime}$ \\
$W_{1,-1}$ & $x^{3} z^{2}+y^{2}+z^{4}+x^{5}$ & IIB & $x^{3} z^{2}+y^{2}+y z^{2}-x^{4} z$ & $K_{10}^{\prime}$ \\
$W_{1,-1}^{\sharp}$ & $\left(x^{3}+z^{2}\right)^{2}+y^{2}+x^{4} z$ & IIB $^{\sharp}$ & $x^{3} y+y^{2}+y z^{2}-x^{4} z$ & $L_{10}$ \\
$S_{1,-1}$ & $x y^{2}+x^{2} z^{2}+y z^{2}+x^{4}$ & IV $_{2}$ & $x y^{2}+x^{2} z^{2}+y z^{2}-x^{3} z$ & $K_{11}^{\prime}$ \\
$S_{1,-1}^{\sharp}$ & $x^{3} y+x y^{2}+y z^{2}+x^{3} z$ & IV $_{2}^{\sharp}$ & $x^{3} y+x y^{2}+y z^{2}-x^{3} z$ & $L_{11}$ \\
$U_{1,-1}$ & $x^{2} y+y^{3}+y z^{3}+x^{2} z$ & IV $_{2}^{\sharp}$ & $x^{2} y+x y^{2}+y z^{3}-x^{2} z$ & $M_{11}$ \\
\hline
\end{tabular}

$\mathbf{h}$ has besides the origin an additional critical point which is of type $A_{1} \cdot{ }^{1}$ This also gives an explanation of the deformation $\mathbf{h}(x, y, z)-x y z$ which we used to define the Gabrielov numbers.

Example 8 We consider the case $J_{3,-1}$ and the 1-parameter family $\mathbf{h}(x, y, z)-t \cdot x y z$, $t \in \mathbb{C}$, where $\mathbf{h}(x, y, z)=x^{3}+x^{2} y^{3}+z^{2}-y^{4} z$. A comparison with Ebeling and Takahashi (2011, Table 12) shows that for $t=0$ the germ at the origin is the exceptional unimodal singularity $E_{14}$. For $t \neq 0$ the substitution $x \mapsto x-\frac{1}{t} y^{3}$ yields

$$
\mathbf{h}\left(x-\frac{1}{t} y^{3}, y, z\right)-t \cdot\left(x-\frac{1}{t} y^{3}\right) y z
$$

\footnotetext{
${ }^{1}$ Note that this is different for some of the original equations of Arnold. There in the cases $W_{1,-1}, S_{1,-1}$, and $U_{1,-1}$ we have two additional critical points and the singularities $W_{12}, S_{11}$, and $Q_{11}$ respectively at the origin.
} 
Table 11 Strange duality of virtual bimodal singularities and ICIS

\begin{tabular}{lllllc}
\hline Name & $\operatorname{Dol}(\mathbf{h})$ & $\operatorname{Gab}(\mathbf{h})$ & $\operatorname{Dol}\left(\widetilde{\mathbf{f}}_{1}, \widetilde{\mathbf{f}}_{2}\right)$ & $\operatorname{Gab}\left(\widetilde{\mathbf{f}}_{1}, \widetilde{\mathbf{f}}_{2}\right)$ & Dual \\
\hline$J_{3,-1}$ & 2,$2 ; 2,3$ & $2,3,10$ & $2,3,10$ & 2,$2 ; 2,3$ & $J_{9}^{\prime}$ \\
$Z_{1,-1}$ & 2,$2 ; 2,4$ & $2,4,8$ & $2,4,8$ & 2,$2 ; 2,4$ & $J_{10}^{\prime}$ \\
$Q_{2,-1}$ & 2,$2 ; 2,5$ & $3,3,7$ & $3,3,7$ & 2,$2 ; 2,5$ & $J_{11}^{\prime}$ \\
$W_{1,-1}$ & 2,$2 ; 3,3$ & $2,6,6$ & $2,6,6$ & 2,$2 ; 3,3$ & $K_{10}^{\prime}$ \\
$W_{1,-1}^{\sharp}$ & 2,$3 ; 2,3$ & $2,5,7$ & $2,5,7$ & 2,$3 ; 2,3$ & $L_{10}$ \\
$S_{1,-1}$ & 2,$2 ; 3,4$ & $3,5,5$ & $3,5,5$ & 2,$2 ; 3,4$ & $K_{11}^{\prime}$ \\
$S_{1,-1}^{\sharp}$ & 2,$3 ; 2,4$ & $3,4,6$ & $3,4,6$ & 2,$3 ; 2,4$ & $L_{11}$ \\
$U_{1,-1}$ & 2,$3 ; 3,3$ & $4,4,5$ & $4,4,5$ & 2,$3 ; 3,3$ & $M_{11}$ \\
\hline
\end{tabular}

Table 12 Coxeter-Dynkin diagrams of virtual bimodal singularities

\begin{tabular}{llllll}
\hline Virtual & Equation & Germ at 0 & Numbers $M_{j}$ & $\gamma_{1}, \gamma_{2}, \gamma_{3}$ & $\mu$ \\
\hline$J_{3,-1}$ & $x^{2}+y^{3}+y^{2} z^{3}-x z^{4}$ & $E_{14}$ & $7+1,7$ & $2,3,9+1$ & 15 \\
$Z_{1,-1}$ & $x^{2}+y^{3}(z-y)+y^{2} z^{3}-x z^{3}$ & $Z_{13}$ & $5+1,3,5$ & $2,4,7+1$ & 14 \\
$Q_{2,-1}$ & $x^{3}+(z-x) y^{2}+x^{2} z^{2}-y z^{3}$ & $Q_{12}$ & $2,2,4+1,4$ & $3,3,6+1$ & 13 \\
$W_{1,-1}$ & $x^{2} y+y^{2}+x^{2} z^{3}-x z^{4}$ & $W_{13}$ & $5,4+1,4$ & $2,5+1,6$ & 14 \\
$W_{1,-1}^{\sharp}$ & $x^{2} y+y^{2}+y z^{3}-x z^{4}$ & $W_{13}$ & $5+1,4,4$ & $2,5,6+1$ & 14 \\
$S_{1,-1}$ & $x^{2} y+(z-y) y^{2}+x^{2} z^{2}-x z^{3}$ & $S_{12}$ & $2,4,3+1,3$ & $3,4+1,5$ & 13 \\
$S_{1,-1}^{\sharp}$ & $x^{2} y+(z-y) y^{2}+y z^{3}-x z^{3}$ & $S_{12}$ & $2,4+1,3,3$ & $3,4,5+1$ & 13 \\
$U_{1,-1}$ & $x^{2} y+x y^{2}+y z^{3}-x^{2} z$ & $S_{12}$ & $2+1,4,3,3$ & $3+1,4,5$ & 13 \\
\hline
\end{tabular}

$$
\begin{aligned}
& =\left(x-\frac{1}{t} y^{3}\right)^{3}+\left(x-\frac{1}{t} y^{3}\right)^{2} y^{3}+z^{2}-y^{4} z-t \cdot\left(x-\frac{1}{t} y^{3}\right) y z \\
& =x^{3}-\frac{3}{t} x^{2} y^{3}+\frac{3}{t^{2}} x y^{6}-\frac{1}{t^{3}} y^{9}+x^{2} y^{3}-\frac{2}{t} x y^{6}+\frac{1}{t^{2}} y^{9}+z^{2}-t \cdot x y z .
\end{aligned}
$$

For $t \neq 0,1$ the coefficient of $y^{9}$ is non-zero. Therefore, the arguments of Example 3 show that in this case the germ at 0 is right equivalent to the cusp singularity $x^{3}+$ $y^{9}+z^{2}-t \cdot x y z$ with Milnor number 13. For $t=1$, the coefficient of $y^{9}$ is equal to zero and Example 3 shows that the germ at 0 is right equivalent to the cusp singularity $x^{3}+y^{10}+z^{2}-x y z$ with Milnor number 14 . One can easily compute that the situation is as follows: For $t \notin\{0,1\}$, the polynomial $\mathbf{h}(x, y, z)-t \cdot x y z$ has two additional critical points of type $A_{1}$ outside the origin. One of them merges with the singularity at the origin for $t=0$, the other one merges with the singularity at the origin for $t=1$.

Now we want to consider Coxeter-Dynkin diagrams of these singularities. Let $X:=\left\{(x, y, z) \in \mathbb{C}^{3} \mid \mathbf{h}(x, y, z)=0\right\}$. The function $\mathbf{h}$ defines a locally trivial fibration $\mathbf{h}: \mathbb{C}^{3} \backslash X \rightarrow \mathbb{C}^{*}$. Let $V=\mathbf{h}^{-1}(1) \cap X$ be the Milnor fibre of this fibration. We shall consider a (strongly) distinguished basis of vanishing cycles of the homology group $\mathrm{H}_{2}(V ; \mathbb{Z}$ ) (see e.g. Arnold et al. 1988; Ebeling 2007). The critical point outside the origin gives an additional vanishing cycle in $H_{2}(V ; \mathbb{Z})$. We define the Milnor number 
(a)

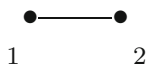

(b)

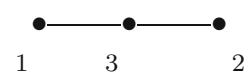

(c)

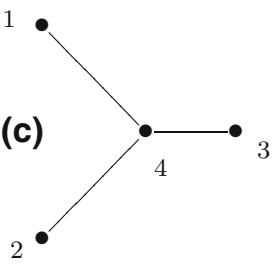

Fig. 1 Coxeter-Dynkin diagrams of a distinguished basis for $\left.\mathbf{h}\right|_{z=0}$

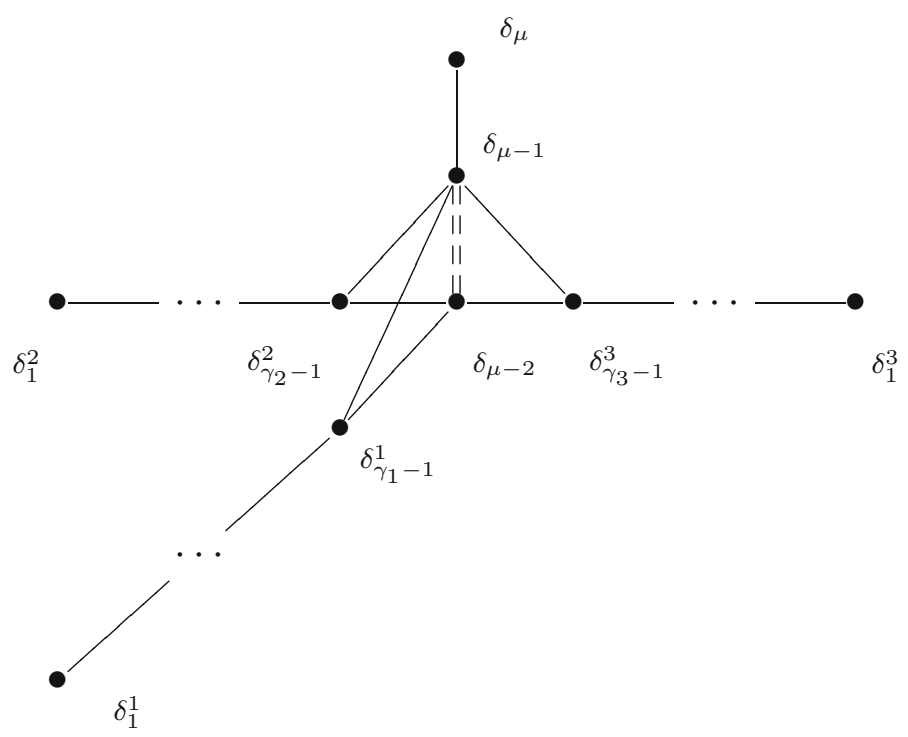

Fig. 2 The graph $S_{\gamma_{1}, \gamma_{2}, \gamma_{3}}$

$\mu$ of $X$ to be the rank of $H_{2}(V ; \mathbb{Z})$. It is equal to the sum of the Milnor numbers of the singular points of $\mathbf{h}$. It is indicated in Table 12.

In order to compute a Coxeter-Dynkin diagram for a distinguished basis of vanishing cycles we use the method of Gabrielov (1979). We have to consider the polar curve corresponding to a choice of a linear function $z: \mathbb{C}^{n} \rightarrow \mathbb{C}$. The choice of the function is indicated in Table 12. The additional critical point lies on the polar curve. One can easily generalize the method of Gabrielov to include this additional critical point. By Gabrielov (1979), one obtains an intersection matrix of a distinguished basis of $\mathbf{h}$ from the one of a distinguished basis for $\left.\mathbf{h}\right|_{z=0}$ by the following formulas. Let $\left(e_{j}\right)(j=1,2$ in case $\mathrm{a}, j=1,2,3$ in case $\mathrm{b}$ and $j=1,2,3,4$ in case c) be a distinguished basis of $\left.\mathbf{h}\right|_{z=0}$ corresponding to the Coxeter-Dynkin diagram presented in Fig. 1. Let $M_{j}$ be the numbers indicated in Table 12. Then there is a distinguished basis $\left(e_{j}^{m}, 1 \leq m \leq M_{j}\right)$ with the following intersection numbers

$$
\left\langle e_{j}^{m}, e_{j^{\prime}}^{m}\right\rangle=\left\langle e_{j}, e_{j^{\prime}}\right\rangle
$$




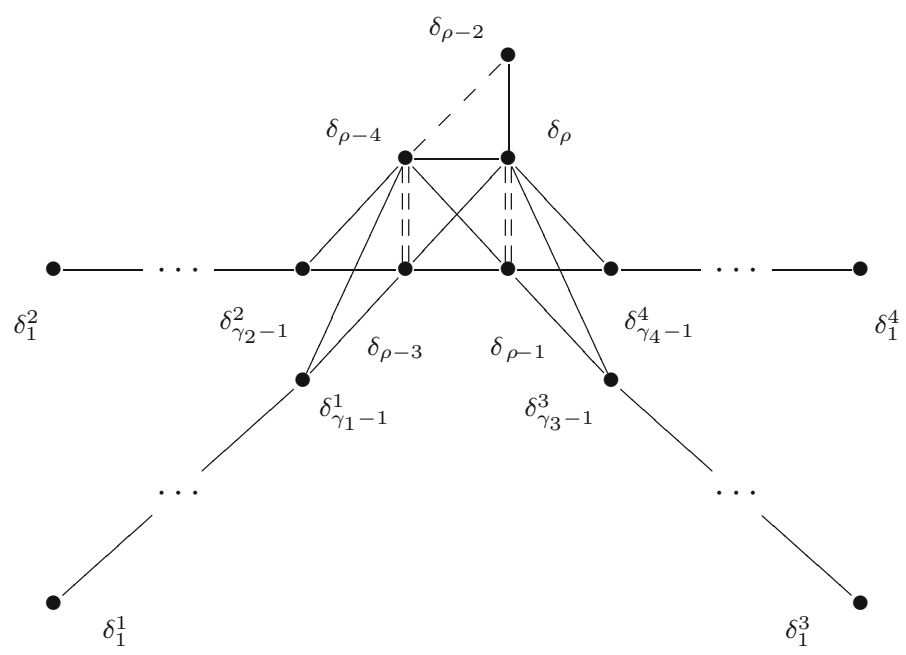

Fig. 3 The graph $\Pi_{\gamma_{1}, \gamma_{2}, \gamma_{3}, \gamma_{4}}$

$$
\begin{aligned}
& \left\langle e_{j}^{m}, e_{j}^{m^{\prime}}\right\rangle=1 \text { for }\left|m^{\prime}-m\right|=1, \\
& \left\langle e_{j}^{m}, e_{j^{\prime}}^{m^{\prime}}\right\rangle=-\left\langle e_{j}, e_{j^{\prime}}\right\rangle \text { for }\left|m^{\prime}-m\right|=1 \text { and }\left(m^{\prime}-m\right)\left(j^{\prime}-j\right)<0, \\
& \left\langle e_{j}^{m}, e_{j^{\prime}}^{m^{\prime}}\right\rangle=0 \text { for }\left|m^{\prime}-m\right|>1 \text { or }\left(m^{\prime}-m\right)\left(j^{\prime}-j\right)>0 .
\end{aligned}
$$

In Table 12, the contribution of the additional critical point to the numbers $M_{j}$ is indicated. By the sequences of elementary basis transformations indicated in Ebeling (1996), the distinguished basis $\left(e_{j}^{m}\right)$ can be transformed to a distinguished basis

$$
\left(\delta_{1}^{1}, \delta_{2}^{1}, \ldots, \delta_{\gamma_{1}-1}^{1} ; \delta_{1}^{2}, \delta_{2}^{2}, \ldots, \delta_{\gamma_{2}-1}^{2} ; \delta_{1}^{3}, \delta_{2}^{3}, \ldots, \delta_{\gamma_{3}-1}^{3} ; \delta_{\mu-2}, \delta_{\mu-1}, \delta_{\mu}\right)
$$

with a Coxeter-Dynkin diagram of the form of Fig. 2 where $\gamma_{1}, \gamma_{2}, \gamma_{3}$ are the Gabrielov numbers of $X$. We call this graph $S_{\gamma_{1}, \gamma_{2} \gamma_{3}}$.

Now let us consider the dual pair $\left(\mathbf{f}_{1}, \widetilde{\mathbf{f}}_{2}\right)$ and the isolated complete intersection singularity defined by it. According to Ebeling (1987, Proposition 3.6.1) one can find a Coxeter-Dynkin diagram with respect to a distinguished basis of thimbles of the form $\Pi_{\gamma_{1}, \gamma_{2}, \gamma_{3}, \gamma_{4}}$ of Fig. 3 where $\gamma_{1}, \gamma_{2} ; \gamma_{3}, \gamma_{4}$ are the Gabrielov numbers of the pair $\left(\widetilde{\mathbf{f}}_{1}, \widetilde{\mathbf{f}}_{2}\right)$.

Acknowledgments We would like to thank the anonymous referee for carefully reading our paper and for useful comments which helped to improve the paper.

\section{References}

Arnold, V.I.: Normal forms of functions in neighbourhoods of degenerate critical points. Uspekhi mat. Nauk 29, 11-49 (1974) (Engl. translation in Russian Math. Surveys 29, 19-48 (1974))

Arnold, V.I.: Critical points of smooth functions and their normal forms. Usp. Math. Nauk. 30(5), 3-65 (1975) (Engl. translation in Russ. Math. Surv. 30(5), 1-75 (1975)) 
Arnold, V.I., Gusein-Zade, S.M., Varchenko, A.N.: Singularities of Differentiable Maps, vol. II. Birkhäuser, Boston (1988)

Berglund, P., Henningson, M.: Landau-Ginzburg orbifolds, mirror symmetry and the elliptic genus. Nucl. Phys. B 433, 311-332 (1995)

Berglund, P., Hübsch, T.: A generalized construction of mirror manifolds. Nucl. Phys. B 393, 377-391 (1993)

Ebeling, W.: The Monodromy Groups of Isolated Singularities of Complete Intersections. Lecture Notes in Mathematics, vol. 1293. Springer, Berlin (1987)

Ebeling, W.: On Coxeter-Dynkin diagrams of hypersurface singularities. J. Math. Sci. 82, 3657-3664 (1996)

Ebeling, W.: Functions of Several Complex Variables and Their Singularities. Graduate Studies in Mathematics, vol. 83. American Mathematical Society, Providence (2007)

Ebeling, W., Gusein-Zade, S.M.: Monodromies and Poincaré series of quasihomogeneous complete intersections. Abh. Math. Sem. Univ. Hambg. 74, 175-179 (2004)

Ebeling, W., Takahashi, A.: Strange duality of weighted homogeneous polynomials. Compos. Math. 147, 1413-1433 (2011)

Ebeling, W., Takahashi, A.: Mirror symmetry between orbifold curves and cusp singularities with group action. Int. Math. Res. Not. 2013, 2240-2270 (2013)

Ebeling, W., Wall, C.T.C.: Kodaira singularities and an extension of Arnold's strange duality. Compos. Math. 56, 3-77 (1985)

Gabrielov, A.M.: Polar curves and intersection matrices of singularities. Invent. math. 54, 15-22 (1979)

Kouchnirenko, A.G.: Polyèdres de Newton et nombres de Milnor. Invent. Math. 32, 1-31 (1976)

Krawitz, M.: FJRW-Rings and Landau-Ginzburg mirror symmetry (2009). arXiv:0906.0796

Libgober, A., Sperber, S.: On the zeta function of monodromy of a polynomial map. Compos. Math. 95, 287-307 (1995)

Saito, K.: Duality for regular systems of weights: a précis. In: Kashiwara, M., Matsuo, A., Saito, K., Satake, I. (eds.) Topological Field Theory, Primitive Forms and Related Topics. Progress in Mathematics, vol. 160, pp. 379-426. Birkhäuser, Boston (1998a)

Saito, K.: Duality for regular systems of weights. Asian J. Math. 2(4), 983-1047 (1998b)

Wall, C.T.C.: Classification of unimodal isolated singularities of complete intersections. Proc. Symp. Pure Math. 40(Part 2), 625-640 (1983) 